\title{
Biomorphic Cellular Silicon Carbide Nanocrystal-Based Ceramics Derived from Wood for use as Thermally Stable and Lightweight Structural Materials
}

Jian-Fei Zhang1, Xiao-Nan Zhou1, Xin Huang1, Liu-Cheng Hao1, 2, Qiang Zhi1, ZiXuan Li1, Bao-Qiang Hou1, Jian-Feng Yang1, Bo Wang1*, Kozo Ishizaki3

1State Key Laboratory for Mechanical Behavior of Materials, Xi'an Jiaotong University, Xi'an710049, China

2 High Voltage Switchgear Insulation Materials Laboratory of State Grid, Pinggao

Electic Co. , Ltd , Pingdingshan 467001, China

3Department of Mechanical Engineering, Nagaoka University of Technology, Nagaoka 940-2188, Japan

*Corresponding author. E-mail: wangbo_1@xjtu.edu.cn 


\section{Supporting Information Available:}

Table S1. Comparison of physical properties and strength in this work with porous

$\mathrm{SiC}$ ceramics in other work.

\begin{tabular}{|c|c|c|c|c|c|}
\hline $\begin{array}{l}\text { The molding } \\
\text { method }\end{array}$ & The sintering method & $\begin{array}{c}\text { Compress } \\
\text { strength } \\
(\mathrm{MPa}) \\
\end{array}$ & $\begin{array}{c}\text { Flexural } \\
\text { strength } \\
\text { (MPa) }\end{array}$ & $\begin{array}{l}\text { Porosity } \\
(\%)\end{array}$ & $\begin{array}{c}\operatorname{Re} \\
\text { f. }\end{array}$ \\
\hline Our work & Vapor SiO infiltration & - & $19 \sim 38$ & $83 \sim 86$ & - \\
\hline \multirow{7}{*}{$\begin{array}{l}\text { Biological } \\
\text { template }\end{array}$} & Liquid Si infiltration & 26 & - & 75 & 1 \\
\hline & Liquid Si infiltration & 8 & - & 72 & 2 \\
\hline & Liquid LPCS infiltration & $32 \sim 37$ & - & $70 \sim 80$ & 3 \\
\hline & $\mathrm{SiO}_{2} \mathrm{Sol}$ infiltration & 7 & 1 & 75 & 4 \\
\hline & $\begin{array}{c}\text { Phenolic resin } / \mathrm{SiO}_{2} \text { sol } \\
\text { infiltration }\end{array}$ & - & $16 \sim 36$ & $54 \sim 76$ & 5 \\
\hline & Si sol infiltration & - & $2 \sim 26$ & $59 \sim 73$ & 6 \\
\hline & Vapor $\mathrm{CH}_{3} \mathrm{SiCl}_{3}$ infiltration & 21 & - & 60 & 7 \\
\hline \multirow{3}{*}{ Freeze casting } & Liquid-phase sintering & 17 & - & 87 & 8 \\
\hline & Solid state sintering & - & $4 \sim 11$ & $66 \sim 72$ & 9 \\
\hline & Pyrolysis & $5 \sim 19$ & - & $59 \sim 85$ & 10 \\
\hline \multirow{4}{*}{$\begin{array}{l}\text { Polymer derived } \\
\text { ceramics }\end{array}$} & Solid sintering & 0.75 & - & 87 & 11 \\
\hline & Solid sintering & $4 \sim 6$ & - & $82 \sim 84$ & 12 \\
\hline & Liquid-phase sintering & $10 \sim 80$ & $8 \sim 25$ & $52 \sim 69$ & 13 \\
\hline & Liquid-phase sintering & - & $18 \sim 50$ & $45 \sim 78$ & 14 \\
\hline \multirow{9}{*}{ Other methods } & Recrystallization sintering & - & $1.5 \sim 5$ & $62 \sim 81$ & 15 \\
\hline & Recrystallization sintering & - & 45 & 47 & 16 \\
\hline & Recrystallization sintering & $11 \sim 28$ & - & $69 \sim 81$ & 17 \\
\hline & Spark plasma sintering & 105 & 26 & 70 & 18 \\
\hline & Adding sintering additive & - & $13 \sim 17$ & $60 \sim 61$ & 19 \\
\hline & Adding foaming agent & - & $8 \sim 10$ & $60 \sim 65$ & 20 \\
\hline & Reaction-bonding & - & $4 \sim 22$ & $33 \sim 52$ & 21 \\
\hline & Reaction-bonding & - & $12 \sim 36$ & $46 \sim 55$ & 22 \\
\hline & Reaction-bonding & - & $5 \sim 28$ & $44 \sim 53$ & 23 \\
\hline
\end{tabular}

Table $\mathbf{S 2}$ shows elemental mass ratio of $\mathrm{C}_{\mathrm{B}}$-template and bio-SiC ceramic sintered at $1800{ }^{\circ} \mathrm{C}$. The elementary composition was determined by a X-ray fluorescence spectroscopy (Bruker S8 Tiger, Germany). The mineral impurities of $\mathrm{C}_{\mathrm{B}}$-template was primarily $\mathrm{Ca}, \mathrm{K}, \mathrm{Si}$ and $\mathrm{Mg}$, which contain up to $2 \%$ (wt). After $\mathrm{SiO}$ vapor infiltration, 
the content of mineral impurities decrease to $0.3 \%(\mathrm{wt})$.

Table S2. Elemental analysis $(\mathrm{XRF})$ of $\mathrm{C}_{\mathrm{B}}$-template and bio-SiC ceramic sintered at $1800{ }^{\circ} \mathrm{C}$.

\begin{tabular}{ccc}
\hline Element & $\mathbf{C}_{\mathbf{B}}$-template $(\mathbf{w t} \%)$ & SiC (wt\%) \\
\hline $\mathrm{Si}$ & 0.18 & 68.58 \\
$\mathrm{Ca}$ & 1.21 & 0.09 \\
$\mathrm{Mg}$ & 0.06 & 0.07 \\
$\mathrm{~K}$ & 0.77 & 0.15 \\
\hline
\end{tabular}

\section{References:}

[1] F.M. Varela-Feriaa; J. Martı'nez-Ferna'ndeza; A.R. de Arellano-Lo' peza; M. Singh. Low Density Biomorphic Silicon Carbide: Microstructure and Mechanical Properties. J Eur Ceram Soc. 2002, 22, 2719-2725.

[2] Gómez-Martín, A.; Orihuela, M. P.; Becerra, J. A.; Martínez-Fernández, J.; Ramírez-Rico, J., Permeability and Mechanical Integrity of Porous Biomorphic Sic Ceramics for Application as Hot-Gas Filters. Materials \& Design 2016, 107, 450-460.

[3] Wang, X.; Liu, J.; Hou, F.; Lu, X.; Sun, X.; Zhou, Y., Manufacture of Porous SiC/C Ceramics with Excellent Damage Tolerance by Impregnation of Lpcs into Carbonized Pinewood. J Eur Ceram Soc 2015, 35 (6), 1751-1759.

[4] Herzog, A; Klingner R; Vogt U; Graule T. Wood-Derived Porous SiC Ceramics by Sol Infiltration and Carbothermal Reduction. J Am Ceram Soc 2004, 87 (5), 784-793.

[5] Qian, J.-M.; Jin, Z.-H., Preparation and Characterization of Porous, Biomorphic Sic Ceramic with Hybrid Pore Structure. J Eur Ceram Soc 2006, 26 (8), 1311-1316. 
[6] Herzog, A.; Vogt, U.; Kaczmarek, O.; Klingner, R.; Richter, K.; Thoemen, H., Porous Sic Ceramics Derived from Tailored Wood-Based Fiberboards. J Am Ceram Soc 2006, 89 (5), 1499-1503.

[7] Greila, P; Voglia, E; Feya, T; Bezolda, A; Popovskab, N; Gerharda, H; Siebera, H. Effect of Microstructure on the Fracture Behavior of Biomorphous Silicon Carbide Ceramics. 2002, 22(14-15), 2697-2707.

[8] Fukushima, M.; Nakata, M.; Zhou, Y.; Ohji, T.; Yoshizawa, Y.-i., Fabrication and Properties of Ultra Highly Porous Silicon Carbide by the Gelation-Freezing Method. $J$ Eur Ceram Soc 2010, 30 (14), 2889-2896.

[9] Wang, F.; Yao, D.; Xia, Y.; Zuo, K.; Xu, J.; Zeng, Y., Porous Sic Ceramics Prepared Via Freeze-Casting and Solid State Sintering. Ceram Int 2016, 42 (3), 45264531.

[10] Xue, F.; Zhou, K.; Wu, N.; Luo, H.; Wang, X.; Zhou, X.; Yan, Z.; Abrahams, I.; Zhang, D., Porous Sic Ceramics with Dendritic Pore Structures by Freeze Casting from Chemical Cross-Linked Polycarbosilane. Ceram Int 2018, 44 (6), 6293-6299.

[1] Jana, P.; Zera, E.; Sorarù, G. D., Processing of Preceramic Polymer to Low Density Silicon Carbide Foam. Materials \& Design 2017, 116, 278-286.

[12] Simonenko, E. P.; Simonenko, N. P.; Zharkov, M. A.; Shembel, N. L.; SimonovEmel'yanov, I. D.; Sevastyanov, V. G.; Kuznetsov, N. T., Preparation of High-Porous Sic Ceramics from Polymeric Composites Based on Diatomite Powder. Journal of Materials Science 2014, 50 (2), 733-744.

[13] Eom, J.-H.; Kim, Y.-W.; Song, I.-H.; Kim, H.-D., Processing and Properties of 
Polysiloxane-Derived Porous Silicon Carbide Ceramics Using Hollow Microspheres as Templates. J Eur Ceram Soc 2008, 28 (5), 1029-1035.

[14] Chae, S.-H.; Kim, Y.-W.; Song, I.-H.; Kim, H.-D.; Narisawa, M., Porosity Control of Porous Silicon Carbide Ceramics. J Eur Ceram Soc 2009, 29 (13), 2867-2872.

[15] Liu, G.; Dai, P.; Wang, Y.; Yang, J.; Qiao, G., Fabrication of Pure Sic Ceramic Foams Using Sio2 as a Foaming Agent Via High-Temperature Recrystallization. Materials Science and Engineering: A 2011, 528 (6), 2418-2422.

[16] Kim, Y.; Min, K.; Shim, J.; Kim, D. J., Formation of Porous Sic Ceramics Via Recrystallization. J Eur Ceram Soc 2012, 32 (13), 3611-3615.

[17] Ganesh, I.; Jana, D. C.; Shaik, S.; Thiyagarajan, N., An Aqueous Gelcasting Process for Sintered Silicon Carbide Ceramics. J Am Ceram Soc 2006, 89 (10), 30563064.

[18] Chen, W.; Miyamoto, Y., Fabrication of Porous Silicon Carbide Ceramics with High Porosity and High Strength. J Eur Ceram Soc 2014, 34 (3), 837-840.

[19] Chi, W.; Jiang, D.; Huang, Z.; Tan, S., Sintering Behavior of Porous Sic Ceramics. Ceram Int 2004, 30 (6), 869-874.

[20] Wan, L.; Tian, Q.; Xiang, Y.; Chen, L.; Song, J.; Wang, X.; Guo, X., Preparation of Porous Sic-A12o3 Ceramics Via Gelcasting Utilising a Shrinkable Pore-Forming Agent and Oxidised Coarse-Grained Sic. Ceram Int 2019, 45 (5), 5511-5517.

[21] Han, F.; Zhong, Z.; Yang, Y.; Wei, W.; Zhang, F.; Xing, W.; Fan, Y., High Gas Permeability of Sic Porous Ceramics Reinforced by Mullite Fibers. J Eur Ceram Soc 2016, 36 (16), 3909-3917. 
[22] Kennedy, G. P.; Lim, K.-Y.; Kim, Y.-W.; Song, I.-H.; Kim, H.-D., Effect of Sic Particle Size on Flexural Strength of Porous Self-Bonded Sic Ceramics. Metals and Materials International 2011, 17 (4), 599-605.

[23] Ding, S.; Zhu, S.; Zeng, Y.; Jiang, D., Effect of Y2o3 Addition on the Properties of Reaction-Bonded Porous Sic Ceramics. Ceram Int 2006, 32 (4), 461-466. 